\title{
Concentrations of fetuin-A, osteoprotegerin and $\alpha$-Klotho in patients with alcoholic liver cirrhosis
}

\author{
ANDRZEJ PRYSTUPA ${ }^{1}$, ANNA DĄBROWSKA ${ }^{2}$, JAROSŁAW JERZY SAK ${ }^{3,4}$, JERZY TARACH $^{2}$, \\ ANNA TORUŃ-JURKOWSKA ${ }^{5}$, PATRYCJA LACHOWSKA-KOTOWSKA ${ }^{1}$ and GRZEGORZ DZIDA ${ }^{1}$ \\ Departments of ${ }^{1}$ Internal Medicine, ${ }^{2}$ Endocrinology, ${ }^{3}$ Nephrology, ${ }^{4}$ Ethics and Human Philosophy and \\ ${ }^{5}$ Mathematics and Medical Biostatistics, Medical University of Lublin, 20-059 Lublin, Poland
}

Received May 29, 2016; Accepted July 26, 2016

DOI: 10.3892/etm.2016.3754

\begin{abstract}
The aim of the present study was to evaluate the concentrations of fetuin-A, osteoprotegerin (OPG) and $\alpha$-Klotho protein in patients with alcoholic cirrhosis at different stages of the disease, and to demonstrate that fetuin-A, osteoprotegin and $\alpha$-Klotho may be used as markers of the severity of cirrhosis. A total of 54 patients with alcoholic liver cirrhosis treated in various hospitals in the Lublin region of Poland were randomly enrolled. The control group consisted of 18 healthy individuals without liver disease, who did not drink alcohol. Serum levels of fetuin-A, OPG and $\alpha$-Klotho were measured by ELISA kits. Levels of fetuin-A were significantly reduced in patients with alcoholic liver cirrhosis compared with the control group. OPG levels were higher in patients with alcoholic liver cirrhosis than in the controls, whereas the levels of $\alpha$-Klotho were comparable in the cirrhosis and control groups. No statistically significant differences in the concentrations of fetuin-A, OPG and $\alpha$-Klotho protein were demonstrated according to type of liver cirrhosis. The findings of the present study revealed a significant negative correlation between the level of $\alpha$-Klotho protein and C-reactive protein in the patients with alcoholic liver cirrhosis. Concentrations of fetuin-A were lower, whereas those of OPG were higher, in the alcoholic liver cirrhosis group compared with the control group. Fetuin-A, OPG and $\alpha$-Klotho may not be good indicators of liver cirrhosis severity. In conclusion, fetuin-A and OPG may be used in the diagnosis of liver cirrhosis.
\end{abstract}

Correspondence to: Professor Jarosław Jerzy Sak, Department of Ethics and Human Philosophy, Medical University of Lublin, Staszica 4/6 (Collegium Maximum), 20-059 Lublin, Poland E-mail: jareksak@tlen.pl

Abbreviations: OPG, osteoprotegerin; TNFR, tumor necrosis factor receptor; P-Ch, Child-Pugh score; HGF, hepatocyte growth factor; SF, scatter factor; TNF, tumor necrosis factor; IL, interleukin; HCC, hepatocellular carcinoma

Key words: fetuin-A, osteoprotegerin, $\alpha$-Klotho, alcoholic liver cirrhosis, Child-Pugh score

\section{Introduction}

Liver cirrhosis is a common hepatic disease, which is characterized by the hyper-accumulation of connective tissue components and hepatic necrosis. Chronic alcohol consumption has been demonstrated to be a major factor that leads to the development of hepatic cirrhosis. In patients with liver cirrhosis, there is high coincidence of portal hypertension, esophageal varices, and hypoalbuminaemia. Peptide growth factors and cytokines have a pertinent role in the pathogenesis of liver cirrhosis (1).

Fetuin-A is a glycoprotein secreted by the liver, kidneys and choroid plexus, which has been associated with calcification and fibrosis in rat and human studies $(2,3)$. Fetuin A, an extracellular inhibitor of transforming growth factor $\beta$ (4), is a profibrogenic stimulus in liver disease (5). Circulating fetuin-A may be a beneficial serum biomarker in the detection of liver and vascular fibrosis progression in patients with non-alcoholic fatty liver disease (6).

Osteoprotegerin (OPG), a secretory glycoprotein belonging to the tumor necrosis factor (TNF) receptor superfamily, exhibits pleiotropic effects on inflammation, endocrine function and the immune system. Serum OPG concentrations may serve as a noninvasive biomarker to identify patients with nonalcoholic steatohepatitis (7). OPG inhibits the recruitment, proliferation and activation of osteoclasts and has a role in the regulation of bone mass, acting as a soluble factor $(8,9)$. $\alpha$-Klotho protein is a $130 \mathrm{kDa}$, one-transmembrane protein and its expression is confirmed in the kidneys and the parathyroid glands; $\alpha$-Klotho is a key regulator of mineral homeostasis and bile acid/cholesterol metabolism $(10,11)$. Limited studies have been conducted to investigate serum Fetuin-A, OPG and $\alpha$-Klotho protein concentrations in patients with alcoholic liver cirrhosis $(12,13)$.

The aim of the present study was to evaluate the concentrations of fetuin-A, OPG and $\alpha$-Klotho protein in patients with alcoholic cirrhosis in different stages of the disease in order to determine whether these glycoproteins may be used as markers of the severity of cirrhosis.

\section{Materials and methods}

Patients with alcoholic liver cirrhosis treated in various hospitals of the Lublin region were randomly enrolled in the 
present study. The Bioethics Committee at Medical University of Lublin, Poland approved the protocol of the study. The study group consisted of 40 male (74\%) and 14 female (26\%) patients. All patients presented a history of heavy alcohol consumption in absence of positivity for serological viral markers. The stages of cirrhosis were assessed using the Child-Turcotte-Pugh criteria as Child-Pugh scores (P-Ch) A, B and C. All patients exhibited normal serum calcium levels and none had previously received treatment with agents that may potentially have influenced bone metabolism. The control group consisted of 18 healthy individuals who did not have liver disease or drink alcohol. All patients gave their written consent. Characteristics of the study population are presented in Tables I and II. Cases and controls were age- and gender-matched.

Liver cirrhosis diagnosis was based on clinical features, laboratory tests, abdominal ultrasound imaging and history of heavy alcohol consumption.

The tissue sample used in the present study was peripheral blood obtained from the ulnar vein. Blood samples $(7 \mathrm{ml})$ were collected into clot tubes between 08:00 and 10:00 following an 8-12 h overnight fast. Serum was separated by centrifugation for of $10 \mathrm{~min}$ at $1,300 \mathrm{RCF}$ at room temperature, aliquoted and stored at $-20^{\circ} \mathrm{C}$ prior to analysis.

Fetuin-A. Serum fetuin-A concentration was determined using a human fetuin-A ELISA kit (Epitope Diagnositics, Inc., San Diego, CA, USA; cat. no. KT-800), according to the manufacturer's protocol. The assay utilized the two-site 'sandwich' technique with two selected goat anti-human polyclonal fetuin-A antibodies that bind to different epitopes of human fetuin-A. Assay sensitivity was $5.0 \mathrm{ng} / \mathrm{ml}$.

$O P G$. Serum total OPG concentration was determined using a human OPG enzyme immunoassay kit (Quidel Corporation, San Diego, CA, USA; cat. no. 8034), according to the manufacturer's protocol. According to the immunocapture technique, murine monoclonal anti-human OPG and biotin-labeled polyclonal anti-human OPG antibodies were used. Assay sensitivity was $0.4 \mathrm{pmol} / \mathrm{l}$.

$\alpha$-Klotho protein. Serum $\alpha$-Klotho concentration was determined using a human soluble $\alpha$-Klotho assay kit (Immuno-Biological Laboratories; Tecan Group, Ltd., Männedorf, Switzerland; cat. no. JP27998), according to the manufacturer's protocol. The kit utilizes solid phase sandwich ELISA using two types of highly specific antibodies. Tetramethylbenzidine was used as a coloring agent. Assay sensitivity was $6.15 \mathrm{pg} / \mathrm{ml}$.

Biochemical parameters. Biochemical parameters, including aspartate transaminase (AST), alanine transaminase (ALT), bilirubin, albumin, C-reactive protein (CRP) and platelets (PLT) were measured. Serum AST (ASTL; cat. no. 20764949322), ALT (ALTL; cat. no. 20764957322), bilirubin (bilirubin; cat. no. 05795397190), albumin (Albumin Gen 2; cat. no. 03183688122), CRP (CRPLX; cat. no. 2076930322) were measured using the described kits and a COBAS 6000 Clinical Chemistry Analyzer (all Roche Diagnostics, Basel, Switzerland). PLT count was calculated using Fluorocell PLT (cat. no. CD994563) and an XN-2000 hematology autoanalyzer (both Sysmex Corporation, Kobe, Japan). Normal laboratory reference ranges were used for comparison.

Statistical analysis. Measurable variables were characterized with arrhythmic means (M) and standard deviation. Frequencies of occurrence were given for qualitative variables (number and percentage). Measurable variables were assigned qualitative categories.

Prior to calculations, the distribution of measurable variables was evaluated using the K-S and Lilliefors test and the Shapiro-Wilk test, whereas homogeneity of variances was tested via the Brown-Forsythe test. Based on P-values, the lack of normal distribution and/or homogeneity of variances were determined. Differences in the variables analyzed were calculated using the Mann-Whitney or Kruskal-Wallis tests. Inter-variable correlations were checked using the Spearman correlation coefficient. $\mathrm{P}<0.05$ was considered to indicate a statistically significant difference.

Inter-group differences in the concentrations of fetuin, OPG and $\alpha$-Klotho were calculated using a non-parametric Mann-Whitney U test. The effects of biochemical parameters, including AST, ALT, bilirubin, albumin, CRP and PLT, on the levels of fetuin-A, OPG and $\alpha$-Klotho in patients with alcoholic liver cirrhosis were analyzed using the Spearman rank correlation test. Fetuin-A, OPG- and $\alpha$-Klotho-dependent variables and an independent variable 'group', which divides patients with alcoholic liver cirrhosis into $\mathrm{A}, \mathrm{B}$ and $\mathrm{C}$ types, and a control group of healthy individuals without liver cirrhosis, were compared using the Kruskal-Wallis rank test, which is a non-parametric equivalent of analysis of variance.

\section{Results}

The findings of the present study study showed that the concentration of fetuin-A in the control group was significantly increased $(110.00 \mathrm{ng} / \mathrm{ml})$, as compared with patients with alcoholic cirrhosis $(3.50 \mathrm{ng} / \mathrm{ml} ; \mathrm{P}<0.001)$. In contrast, serum OPG concentration was significantly increased in patients with alcoholic liver cirrhosis $(7.49 \mathrm{pmol} / \mathrm{l})$, as compared with control patients $(2,46 \mathrm{pmol} / \mathrm{l} ; \mathrm{P}<0.001)$. $\alpha$-Klotho protein concentration in patients with alcoholic cirrhosis (794.85) was not significantly different from the control group (671.1; $\mathrm{P}>0.05)$. These results are shown in Table III. Moreover, the associations between fetuin-A, OPG and $\alpha$-Klotho concentrations and the stages of cirrhosis according to $\mathrm{P}-\mathrm{Ch}$ scores were analyzed.

Fetuin-A concentration was significantly higher in the control group compared to patients with alcoholic liver cirrhosis at stages $\mathrm{A}, \mathrm{C}(\mathrm{P}<0.001)$ and $\mathrm{B}(\mathrm{P}<0.05)$. No significant differences in the concentrations of fetuin-A were demonstrated according to the type of liver cirrhosis. However, it should be noted that the lowest fetuin-A levels were detected in subjects with the most severe liver cirrhosis (P-Ch C: $65.00 \mathrm{ng} / \mathrm{ml}$ compared with P-Ch A: $77.50 \mathrm{ng} / \mathrm{ml}$ and P-Ch B: $67.50 \mathrm{ng} / \mathrm{ml}$, respectively; (Fig. 1).

Further analysis demonstrated significantly increased concentrations of OPG in patients with alcoholic liver cirrhosis at stages $\mathrm{B}, \mathrm{C}(\mathrm{P}<0.001)$ and $\mathrm{A}(\mathrm{P}<0.05)$ as 
Table I. Characteristics of patients with alcoholic liver cirrhosis and healthy controls.

\begin{tabular}{|c|c|c|c|c|}
\hline Characteristic & Control $(n=18)$ & P-Ch A $(n=14)$ & P-Ch B $(n=20)$ & $\mathrm{P}-\mathrm{Ch} C(\mathrm{n}=20)$ \\
\hline Age (years) & $55.51 \pm 8.89$ & $52.50 \pm 16.11$ & $54.00 \pm 12.19$ & $50.71 \pm 10.00$ \\
\hline Body weight (kg) & $75.63 \pm 9.83$ & $66.33 \pm 11.93$ & $84.84 \pm 27.11$ & $85.91 \pm 21.76$ \\
\hline Height (cm) & $173.54 \pm 10.31$ & $171.33 \pm 9.86$ & $177.36 \pm 11.40$ & $175.45 \pm 6.69$ \\
\hline Drinking period (years) & - & $11.16 \pm 7.40$ & $13.86 \pm 7.06$ & $18.17 \pm 10.73$ \\
\hline \multicolumn{5}{|l|}{ Existing symptoms } \\
\hline Ascites & 0 & 1 & 14 & 15 \\
\hline Encephalopathy & 0 & 3 & 8 & 18 \\
\hline Oesophageal varices & 0 & 2 & 9 & 16 \\
\hline
\end{tabular}

Data are presented as the mean \pm standard deviation. P-Ch, Child-Pugh score.

Table II. Biochemical data of the study participants.

\begin{tabular}{|c|c|c|c|c|}
\hline Variable & Control $(n=18)$ & P-Ch A $(n=14)$ & P-Ch B $(n=20)$ & P-Ch C (n=20) \\
\hline Bilirubin (mg/dl) & $0.64 \pm 0.22$ & $2.70 \pm 0.95$ & $12.31 \pm 4.48$ & $15.75 \pm 4.87$ \\
\hline Albumin (g/dl) & $5.23 \pm 0.54$ & $4.00 \pm 0.67$ & $3.80 \pm 0.84$ & $2.42 \pm 0.48$ \\
\hline $\operatorname{ALT}(\mathrm{U} / \mathrm{l})$ & $19.24 \pm 8.56$ & $99.00 \pm 221.00$ & $41,57 \pm 29,48$ & $61.24 \pm 104.60$ \\
\hline AST (IU/l) & $17.81 \pm 5.03$ & $143.00 \pm 249.0$ & $96.67 \pm 101.57$ & $132.00 \pm 202,06$ \\
\hline GGTP (IU/1) & $20.40 \pm 8.96$ & $313.75 \pm 27.96$ & $642.24 \pm 70.04$ & $749.48 \pm 72.55$ \\
\hline Urea (mg/dl) & $24.40 \pm 10.07$ & $38.77 \pm 6.98$ & $44.81 \pm 8.54$ & $51.25 \pm 5.39$ \\
\hline Blood platelets $(\mathrm{K} / \mathrm{ul})$ & $340.2 \pm 7.96$ & $166.75 \pm 11.96$ & $135.46 \pm 12.28$ & $105.33 \pm 7.02$ \\
\hline INR & $1.26 \pm 0.16$ & $1.30 \pm 0.21$ & $1.39 \pm 0.23$ & $2.01 \pm 0.90$ \\
\hline $\operatorname{MCV}(f l)$ & $86.00 \pm 7.26$ & $95.97 \pm 9.36$ & $97.09 \pm 6.27$ & $103.07 \pm 6.09$ \\
\hline $\mathrm{Na}(\mathrm{mmol} / \mathrm{l})$ & $139.50 \pm 3.44$ & $129.75 \pm 10.50$ & $134.05 \pm 4.78$ & $131.85 \pm 8.41$ \\
\hline $\mathrm{K}(\mathrm{mmol} / \mathrm{l})$ & $4.17 \pm 0.32$ & $3.59 \pm 0.42$ & $4.07 \pm 0.77$ & $3.86 \pm 0.60$ \\
\hline Fetuin-A (ng/ml) & $126.92 \pm 51.20$ & $79.00 \pm 13.80$ & $75.77 \pm 30.06$ & $57.62 \pm 31.91$ \\
\hline OPG (pmol/l) & $2.89 \pm 1.46$ & $7.05 \pm 2.20$ & $8.92 \pm 4.41$ & $6.56 \pm 2.22$ \\
\hline$\alpha$-Klotho (pg/ml) & $645.15 \pm 131.30$ & $719.00 \pm 559.00$ & $1110.50 \pm 911.50$ & $1091.40 \pm 774.50$ \\
\hline
\end{tabular}

Data are expressed as mean \pm standard deviation. Normal ranges: bilirubin, 0-1.2 mg/dl; albumin, 3.5-5.20 g/dl; ALT, 5-40 U/I; AST, 5-40 IU/l; GGTP, 11-50 IU/l; urea, 21-43 mg/dl; blood platelets, 120-400 K/ul; INR, 0.86-1.30; MCV, 80-94 fl; K, 3.5-5.1 mmol/1; and Na, $136-145$ mmol/l. P-Ch, Child-Pugh score; ALT, alanine aminotransferase; AST, aspartate aminotransferase; GGTP, gamma-glutamyl transpeptidase; INR, international normalized ratio; MCV, mean cellular volume; Na, sodium; K, potassium; OPG, osteoprotegerin.

Table III. Fetuin A, OPG and $\alpha$-Klotho levels in controls and patients with alcoholic liver cirrhosis.

\begin{tabular}{|c|c|c|c|c|c|}
\hline \multirow[b]{2}{*}{ Marker } & \multicolumn{2}{|r|}{ Study group } & \multirow[b]{2}{*}{$\mathrm{Z}$ test function } & \multirow[b]{2}{*}{ P-value } & \multirow[b]{2}{*}{ Significance } \\
\hline & Control $(n=18)$ & Patients with liver cirrhosis $(\mathrm{n}=54)$ & & & \\
\hline Fetuin-A (ng/ml) & $126.92 \pm 51.00$ & $71.65 \pm 27.89$ & 4.505 & 0.0001 & $\mathrm{P}<0.001$ \\
\hline OPG (pmol/l) & $2.89 \pm 1.46$ & $7.74 \pm 3.48$ & -5.165 & 0.0001 & $\mathrm{P}<0.001$ \\
\hline$\alpha$-Klotho $(\mathrm{pg} / \mathrm{ml})$ & $645.00 \pm 131.00$ & $1004.00 \pm 789.00$ & -0.904 & 0.366 & $\mathrm{P}>0.05$ \\
\hline
\end{tabular}

$\mathrm{Z}$ values were obtained from the Mann-Whitney test. OPG, osteoprotegerin.

compared with the control group. No significant differences in OPG concentrations were observed among the types of liver cirrhosis (Fig. 2).
Furthermore, no statistically significant differences in $\alpha$-Klotho concentrations were demonstrated between the control and liver cirrhosis groups, or among stages A, B and 


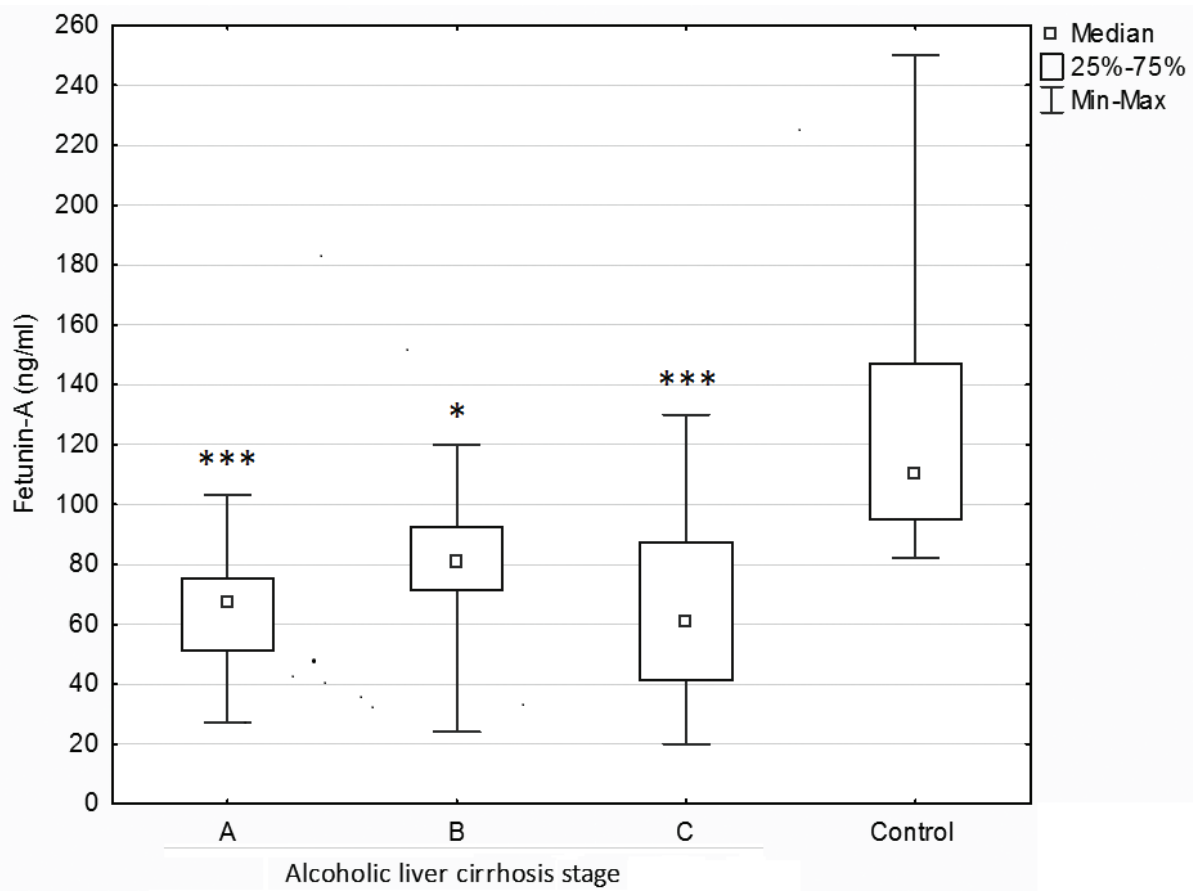

Figure 1. Fetuin-A concentration levels in patients with stage A, B and C alcoholic liver cirrhosis, and the control. ${ }^{*} \mathrm{P}<0.05$ vs. control and ${ }^{* * *} \mathrm{P}<0.001$ vs. control.

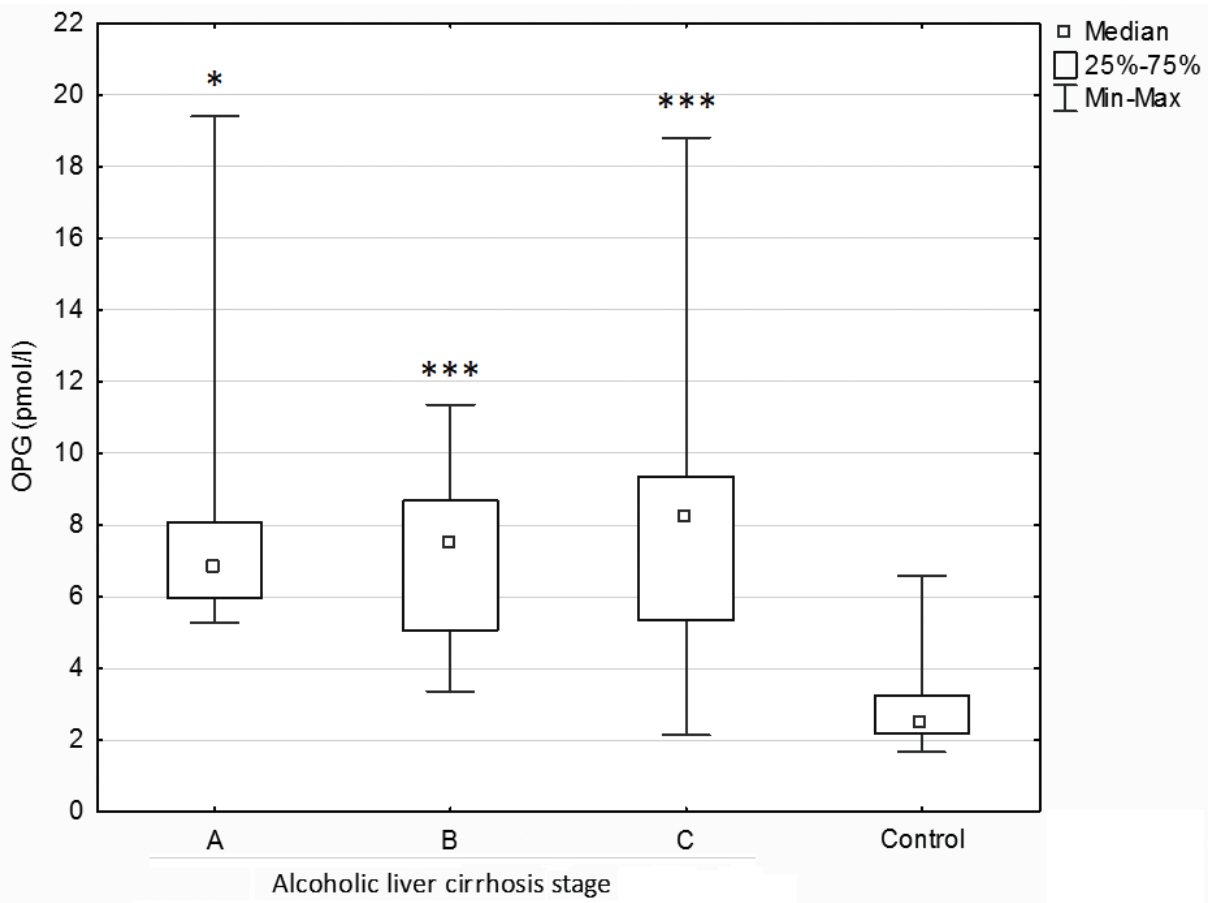

Figure 2. Serum OPG concentration levels in patients with alcoholic cirrhosis at stage A, B, C, and the control. OPG, osteoprotegerin. ${ }^{*} \mathrm{P}<0.05$ vs. control and ${ }^{* * * *} \mathrm{P}<0.001$ vs. control.

C of alcoholic liver cirrhosis (Kruskal-Wallis: $\mathrm{H}=5.491033$ $\mathrm{P}=0.1392$; Fig. 3).

The results revealed a significant negative correlation between the levels of $\alpha$-Klotho and CRP in patients with liver cirrhosis (Fig. 4), indicating that an increase in CRP concentration may lead to a decrease in $\alpha$-Klotho concentration [R Spearman=-0.304; $t(\mathrm{~N}-2)=-2.039 ; \mathrm{P}=0.047]$. No significant correlations were detected for the remaining biochemical variables.

\section{Discussion}

Fetuin-A, which is also referred to as alpha 2-Heremans Schmid glycoprotein, is a multifunctional plasma agent with a molecular weight of $\sim 60 \mathrm{kDa}$ and a half-life of several days that is predominantly secreted by the liver in adults (>95\%) (14). Fetuin-A induces inflammatory cytokine expression and inhibits adiponectin expression. An increase of fetuin A serum levels has been observed in patients with metabolic 


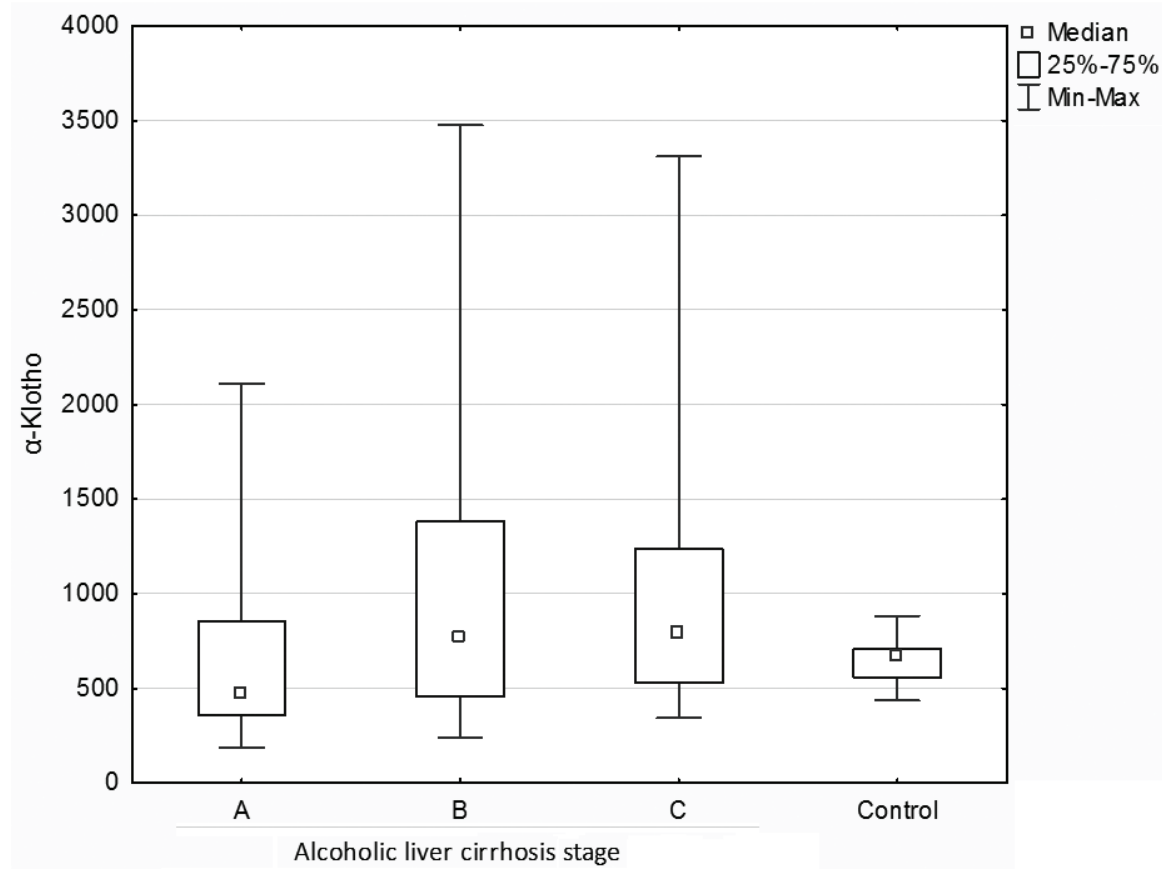

Figure 3. Serum $\alpha$-Klotho concentration levels in patients with alcoholic liver cirrhosis at stage A, B and C and controls.

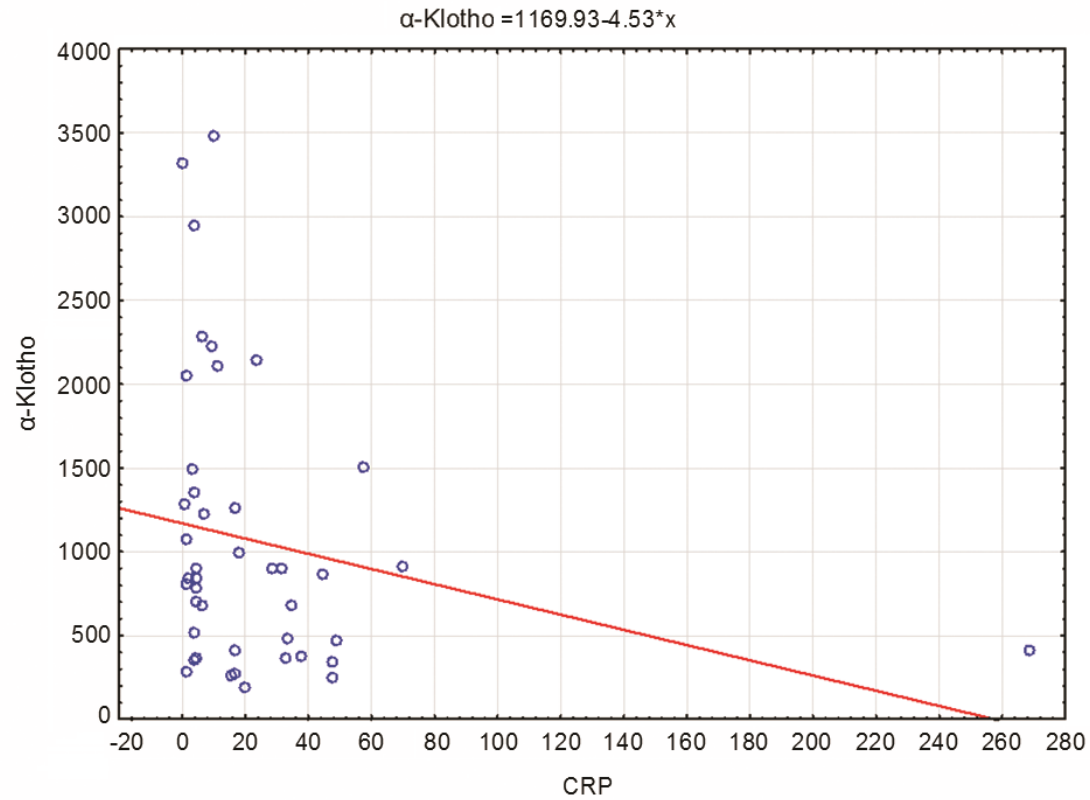

Figure 4. $\alpha$-Klotho protein concentration levels in patients with alcoholic cirrhosis depending on the concentration of CRP. CRP, C-reactive protein.

syndrome, type 2 diabetes and non-alcoholic fatty liver disease (15). Furthermore, fetuin-A has been demonstrated to act as an antagonist of hepatocyte growth factor/scatter factor and as a regulator in tissue regeneration (16), and it may be a relevant biomarker of aortic valve disease (17).

Ohnishi et al (18) indicated that serum fetuin-A concentrations were reduced in alcoholic subjects with liver cirrhosis, which may be a consequence of reduced fetuin-A synthesis by hepatocytes. The present study showed that patients with alcoholic cirrhosis had significantly lower fetuin-A levels than healthy subjects. These results are consistent with previous findings reported by Kalabay et al (19), who demonstrated the same relationship between fetuin-A levels and cirrhosis and hepatocellular carcinoma (HCC). In addition, according to Kalabay et al (13), the tendency to decrease serum fetuin-A concentration is a reliable and sensitive indicator of mortality in patients with alcoholic liver cirrhosis.

Moreover, it has been suggested that decreased serum fetuin-A levels indicate hepatocyte dysfunction rather than an acute phase response (20). This hypothesis may be supported by the lack of correlation between fetuin-A concentration and CRP levels in patients with alcoholic liver cirrhosis in the 
present study. An association between the severity of liver cirrhosis, assessed by P-Ch scores, and fetuin-A levels has not been determined at present. However, the findings of the present study did not reveal a correlation between fetuin-A concentration and the stage of alcoholic liver cirrhosis, and selected laboratory parameters (ALT, ASP, CRP, bilirubin, albumins and PLT). Notably, the lowest fetuin-A levels were detected in subjects with the most severe liver cirrhosis.

Another protein investigated in the present study was OPG. OPG is a glycoprotein member of the TNF ligand family that is coded by a gene located on chromosome 8 , and consists of 380 amino acids. The primary biological action of OPG is the inhibition of osteooclast activity and differentiation from osteooclast precursors (19). In the present study, it was demonstrated that serum OPG levels were significantly increased in patients with alcoholic liver cirrhosis than in healthy subjects. No correlations were detected between the severity of alcoholic liver cirrhosis, according to P-Ch scores (A, B, C) and OPG levels. Previous studies have indicated increased OPG levels in patients with primary biliary and viral cirrhosis $(21,22)$. Furthermore, a rise in glycoprotein concentrations has been reported in subjects with alcoholic liver cirrhosis, in accordance with the results of the present study. In one such report, Fábrega et al (23) reported an increase of OPG levels in 30 patients with cirrhosis compared with 20 controls. Additionally, OPG levels were found to be higher in patients with $\mathrm{P}-\mathrm{Ch} \mathrm{C}$, compared with those with $\mathrm{P}-\mathrm{Ch} \mathrm{A}$. OPG is synthesized in the liver, and expression of OPG mRNA has been detected in hepatocytes, bile duct epithelium, Kupffer cells and lymphocytes (23). Moreover, the inflammatory cells that infiltrate the liver in chronic alcoholic liver disease may have a role in serum elevation of OPG in these subjects. Osteoblasts are another primary source of OPG (24). In patients with liver cirrhosis assessed in previous studies, plasma levels of TNF- $\alpha$, interleukin (IL)-1 and IL-6 were elevated $(25,26)$. OPG is likely to be stimulated by proinflammatory cytokines. Elevated TNF- $\alpha$ and IL- 6 levels are strongly associated with OPG levels (27). TNF- $\alpha$ and IL-6 enhance bone resorption; therefore, their association with OPG suggests a protective effect of raised OPG on bone loss. Gaudio et al (28) have previously shown an increase in OPG levels, possibly in response to enhanced bone loss in patients with liver cirrhosis. The present study indicates that OPG is not a good indicator of the severity of cirrhosis.

The final protein tested in patients with liver cirrhosis was $\alpha$-Klotho, which is essential for mineral metabolism. $\alpha$-Klotho participates in the regulation of parathyroid hormone secretion and vitamin D biosynthesis, in the transepithelial transport of calcium ions in the choroid plexus and kidney, and in renal phosphate re-absorption (28). The association between $\alpha$-Klotho serum concentration and alcoholic liver cirrhosis has not yet been studied.

The results of the present study did not indicate statistically significant differences in $\alpha$-Klotho levels among patients with alcoholic liver cirrhosis and controls. The stage of disease had no effect on $\alpha$-Klotho concentration. Previous studies have characterized $\alpha$-Klotho as an anti-aging hormone that modulates antioxidant enzyme expression levels (29) and has an anti-inflammatory function (30). Therefore, an adequate tissue level of $\alpha$-Klotho may provide protection against oxidative stress and inflammation $(31,32)$. Systemic and local inflammation is associated with decreased renal $\alpha$-Klotho expression levels (33). In the present study, $\alpha$-Klotho concentration was negatively correlated with serum CRP levels in patients with alcoholic liver cirrhosis, as increased levels of $\mathrm{CRP}$ resulted in reduced concentrations of $\alpha$-Klotho. Therefore, it can be speculated that alcoholic liver cirrhosis, which is a chronic inflammatory disorder, may be associated with impaired $\alpha$-Klotho expression and reduced soluble $\alpha$-Klotho concentrations. A similar negative correlation between $\alpha$-Klotho concentration and CRP level has been detected in a study conducted by Navarro-González et al (34). This study showed that $\alpha$-Klotho concentration was reduced in patients with significant coronary artery disease. Furthermore, $\alpha$-Klotho has been revealed to have tumor suppressive properties during various malignant transformations by factor pathway (35), which is associated with cancer risk and tumor progression. Xie et al (36) measured mRNA and protein expression levels of $\alpha$-Klotho in 64 HCC tumor tissues using real-time polymerase chain reaction and immunohistochemistry, respectively, demonstrating that a loss of $\alpha$-Klotho expression in HCC cells was a predictive factor for the poor prognosis of the disease. Similarly, Shu et al (37) reported that exogenous expression of the Klotho gene significantly inhibited the proliferation of HCC cells, induced HCC cell apoptosis and decreased HCC cell migration, using a Matrigel invasion chamber assay. Conversely, following the analysis of 52 hepatoma patients, Chen et al (38) reported that immunohistochemical $\alpha$-Klotho staining was significantly associated with liver cirrhosis, tumor multiplicity and venous invasion, and the survival rate of subjects with high $\alpha$-Klotho expression was significantly decreased compared to patients with low $\alpha$-Klotho expression.

In conclusion, fetuin-A concentration levels are significantly reduced in patients with alcoholic liver cirrhosis compared with controls, whereas $\alpha$-Klotho levels are consistent between the groups. No statistically significant differences in the concentrations of fetuin-A, OPG and $\alpha$-Klotho were demonstrated according to the type of cirrhosis. Furthermore, a significant negative correlation was demonstrated between the levels of $\alpha$-Klotho and CRP in patients with alcoholic liver cirrhosis. These results indicate that fetuin-A, OPG and $\alpha$-Klotho may not be useful markers of the severity of liver cirrhosis, but fetuin-A and ostoprotegerin concentration levels may be used as biomarkers of alcoholic liver cirrhosis.

\section{Acknowledgements}

This study was supported by the Medical University of Lublin, Poland (grant no. DS507).

\section{References}

1. Prystupa A, Szpetnar M, Boguszewska-Czubara A, Grzybowski A, Sak J and Załuska W: Activity of MMP1 and MMP13 and amino acid metabolism in patients with alcoholic liver cirrhosis. Med Sci Monit 21: 1008-1014, 2015.

2. Schinke T, Amendt C, Trindl A, Pöschke O, Müller-Esterl W and Jahnen-Dechent $\mathrm{W}$ : The serum protein alpha2-HS glycoprotein/fetuin inhibits apatite formation in vitro and in mineralizing calvaria cells. A possible role in mineralization and calcium homeostasis. J Biol Chem 271: 20789-20796, 1996. 
3. Karabakan M, Bozkurt A, Gunay M, Aktas BK, Hirik E, Aydın M and Nuhoglu B: Association between serum fetuin-A level and erectile function. Andrologia 48: 787-792, 2016.

4. Swallow CJ, Partridge EA, Macmillan JC, Tajirian T, DiGuglielmo GM, Hay K, Szweras M, Jahnen-Dechent W, Wrana JL, Redston M, et al: Alpha2HS-glycoprotein, an antagonist of transforming growth factor beta in vivo, inhibits intestinal tumor progression. Cancer Res 64: 6402-6409, 2004.

5. Bataller R and Brenner DA: Liver fibrosis. J Clin Invest 115: 209-218, 2005.

6. Sato M, Kamada Y, Takeda Y, Kida S, Ohara Y, Fujii H, Akita M, Mizutani K, Yoshida Y, Yamada M, et al: Fetuin-A negatively correlates with liver and vascular fibrosis in nonalcoholic fatty liver disease subjects. Liver Int 35: 925-935, 2015.

7. Yilmaz Y, Yonal O, Kurt R, Oral AY, Eren F, Ozdogan O, Ari F, Celikel CA, Korkmaz S, Ulukaya E, et al: Serum levels of osteoprotegerin in the spectrum of nonalcoholic fatty liver disease. Scand J Clin Lab Invest 70: 541-546, 2010.

8. Lipton A, Ali SM, Leitzel K, Chinchilli V, Witters L, Engle L, Holloway D, Bekker P and Dunstan CR: Serum osteoprotegerin levels in healthy controls and cancer patients. Clin Cancer Res 8 : 2306-2310, 2002.

9. Kainuma S, Otsuka T, Kuroyanagi G, Yamamoto N, Matsushima-Nishiwaki R, Kozawa O and Tokuda H: Possible involvement of AMP-activated protein kinase in PGE1-induced synthesis of osteoprotegerin in osteoblasts. Exp Ther Med 11: 2042-2048, 2016.

10. Tomiyama K, Maeda R, Urakawa I, Yamazaki Y, Tanaka T, Ito S, Nabeshima Y, Tomita T, Odori S, Hosoda K, et al: Relevant use of Klotho in FGF19 subfamily signaling system in vivo. Proc Natl Acad Sci USA 107: 1666-1671, 2010.

11. Yamazaki Y, Imura A, Urakawa I, Shimada T, Murakami J, Aono Y, Hasegawa H, Yamashita T, Nakatani K, Saito Y, et al: Establishment of sandwich ELISA for soluble alpha-Klotho measurement: Age-dependent change of souble alpha-Klotho levels in healthy subjects. Biochem Biophys Res Commun 398: 513-518, 2010

12. García-Valdecasas-Campelo E, González-Reimers E, Santolaria-Fernández F, De la Vega-Prieto MJ, Milena-Abril A, Sánchez-Pérez MJ, Martínez-Riera A and Gómez-Rodríguez Mde L: Serum osteoprotegerin and RANKL levels in chronic alcoholic liver disease. Alcohol Alcohol 41: 261-266, 2006.

13. Kalabay L, Gráf L, Vörös K, Jakab L, Benko Z, Telegdy L, Fekete B, Prohászka Z and Füst G: Human serum fetuin A/alpha2HS-glycoprotein level is associated with long-term survival in patients with alcoholic liver cirrhosis, comparison with the Child-Pugh and MELD scores. BMC Gastroenterol 7: $15,2007$.

14. Dabrowska AM, Tarach JS, Wojtysiak-Duma B and Duma D Fetuin-A (AHSG) and its usefulness in clinical practice. Review of the literature. Biomed Pap Med Fac Univ Palacky Olomouc Czech Repub 159: 352-359, 2015.

15. Denecke B, Gräber S, Schäfer C, Heiss A, Wöltje M and Jahnen-Dechent W: Tissue distribution and activity testing suggest a similar but not identical function of fetuin-B and fetuin-A. Biochem J 376: 135-145, 2003.

16. Ix JH, Shlipak MG, Brandenburg VM, Ali S, Ketteler M and Whooley MA: Association between human fetuin-A and the metabolic syndrome: Data from the heart and soul study. Circulation 113: 1760-1767, 2006.

17. Zeng YI, Sun R, Li X, Liu M, Chen S and Zhang P: Pathophysiology of valvular heart disease. Exp Ther Med 11: $1184-1188,2016$.

18. Ohnishi T, Nakamura O, Arakaki N and Daikuhara Y: Effect of phosphorylated rat fetuin on the growth of hepatocytes in primary cultures in the presence of human hepatocyte-growth factor. Evidence that phosphorylated fetuin is a natural modulator of hepatocyte-growth factor. Eur J Biochem 243: 753-761, 1997.

19. Kalabay L, Jakab L, Prohászka Z, Füst G, Benkö Z, Telegdy L, Lörincz Z, Závodszky P, Arnaud P and Fekete B: Human fetuin/alpha2HS-glycoprotein level as a novel indicator of liver cell function and short-term mortality in patients with liver cirrhosis and liver cancer. Eur J Gastroenterol Hepatol 14 389-394, 2002
20. Jezequel M, Seta NS, Corbic MM, Feger JM and Durand GM: Modifications of concanavalin A patterns of alpha 1-acid glycoprotein and alpha 2-HS glycoprotein in alcoholic liver disease. Clin Chim Acta 176: 49-57, 1988

21. Hofbauer LC, Khosla S, Dunstan CR, Lacey DL, Boyle WJ and Riggs BL: The roles of osteoprotegerin and osteoprotegerin ligand in the paracrine regulation of bone resorption. $\mathrm{J}$ Bone Miner Res 15: 2-12, 2000.

22. Szalay F, Hegedus D, Lakatos PL, Tornai I, Bajnok E, Dunkel K and Lakatos P: High serum osteoprotegerin and low RANKL in primary biliary cirrhosis. J Hepatol 38: 395-400, 2003.

23. Fábrega E, Orive A, García-Suarez C, García-Unzueta M, Antonio Amado J and Pons-Romero F: Osteoprotegerin and RANKL in alcoholic liver cirrhosis. Liver Int 25: 305-310, 2005.

24. Moschen AR, Kaser A, Stadlmann S, Millonig G, Kaser S, Mühllechner P, Habior A, Graziadei I, Vogel W and Tilg H: The RANKL/OPG system and bone mineral density in patients with chronic liver disease. J Hepatol 43: 973-983, 2005.

25. Kwon BS, Wang S, Udagawa N, Haridas V, Lee ZH, Kim KK, Oh KO, Greene J, Li Y, Su J, et al: TR1, a new member of the tumor necrosis factor receptor superfamily, induces fibroblast proliferation and inhibits osteoclastogenesis and bone resorption. FASEB J 12: 845-854, 1998.

26. Daniluk J, Szuster-Ciesielska A, Drabko J and Kandefer-Szerszeń M: Serum cytokine levels in alcohol-related liver cirrhosis. Alcohol 23: 29-34, 2001

27. Sasso GR, Florencio-Silva R, Simões RS, Baracat MC, Soares Júnior JM and Baracat EC: Elevated serum osteoprotegerin levels in women: Friend or foe? Rev Assoc Med Bras (1992) 61: 524-529, 2015.

28. Gaudio A, Lasco A, Morabito N, Atteritano M, Vergara C, Catalano A, Fries W, Trifiletti A and Frisina N: Hepatic osteodystrophy: Does the osteoprotegerin/receptor activator of nuclear factor-kB ligand system play a role? J Endocrinol Invest 28: 677-682, 2005 .

29. Tsujikawa H, Kurotaki Y, Fujimori T, Fukuda K and Nabeshima Y: Klotho, a gene related to a syndrome resembling human premature aging, functions in a negative regulatory circuit of vitamin D endocrine system. Mol Endocrinol 17: 2393-2403, 2003.

30. Kuro-o M: Klotho as a regulator of oxidative stress and senescence. Biol Chem 389: 233-241, 2008.

31. Zhao Y, Banerjee S, Dey N, LeJeune WS, Sarkar PS, Brobey R, Rosenblatt KP, Tilton RG and Choudhary S: Klotho depletion contributes to increased inflammation in kidney of the $\mathrm{db} / \mathrm{db}$ mouse model of diabetes via RelA (serine) 536 phosphorylation. Diabetes 60: 1907-1916, 2011.

32. Martín-Núñez E, Donate-Correa J, Muros-de-Fuentes M, Mora-Fernández C and Navarro-González JF: Implications of Klotho in vascular health and disease. World J Cardiol 6: 1262-1269, 2014

33. Moreno JA, Izquierdo MC, Sanchez-Niño MD, Suárez-Alvarez B, Lopez-Larrea C, Jakubowski A, Blanco J, Ramirez R, Selgas R, Ruiz-Ortega M, et al: The inflammatory cytokines TWEAK and $\mathrm{TNF} \alpha$ reduce renal klotho expression through NFkB. J Am Soc Nephrol 22: 1315-1325, 2011.

34. Navarro-González JF, Donate-Correa J, Muros de Fuentes M, Pérez-Hernández H, Martínez-Sanz R and Mora-Fernández C: Reduced Klotho is associated with the presence and severity of coronary artery disease. Heart 100: 34-40, 2014.

35. Xie B, Zhou J, Shu G, Liu DC, Zhou J, Chen J and Yuan L: Restoration of klotho gene expression induces apoptosis and autophagy in gastric cancer cells: Tumor suppressive role of klotho in gastric cancer. Cancer Cell Int 13: 18, 2013.

36. Xie B, Zhou J, Yuan L, Ren F, Liu DC, Li Q and Shu G: Epigenetic silencing of Klotho expression correlates with poor prognosis of human hepatocellular carcinoma. Hum Pathol 44: 795-801, 2013.

37. Shu G, Xie B, Ren F, Liu DC, Zhou J, Li Q, Chen J, Yuan L and Zhou J: Restoration of klotho expression induces apoptosis and autophagy in hepatocellular carcinoma cells. Cell Onco (Dordr) 36: 121-129, 2013

38. Chen L, Liu H, Liu J, Zhu Y, Xu L, He H, Zhang H, Wang S, Wu Q, Liu W, et al: Klotho endows hepatoma cells with resistance to anoikis via VEGFR2/PAK1 activation in hepatocellular carcinoma. PLoS One 8: e58413, 2013. 